\title{
Optimization of Nano-Grating Pitch Evaluation Method Based on Line Edge Roughness Analysis
}

\author{
Jie Chen ${ }^{1}$, Jie Liu ${ }^{1}$, Xingrui Wang ${ }^{1}$, Longfei Zhang ${ }^{1}$, Xiao Deng ${ }^{2}$, Xinbin Cheng ${ }^{1}$, Tongbao Li ${ }^{1}$ \\ ${ }^{1}$ Institute of Precision Optical Engineering, School of Physics Science and Engineering, Tongji University, 200092, \\ Shanghai, China \\ ${ }^{2}$ School of Aerospace Engineering and Applied Mechanics, Tongji University, 200092, Shanghai, China \\ Corresponding author: Xiao Deng, Email: 1110490dengxiao@tongji.edu.cn
}

Pitch uncertainty and line edge roughness are among the critical quality attributes of a pitch standard and normally the analyses of these two parameters are separate. The analysis of self-traceable $\mathrm{Cr}$ atom lithography nano-gratings shows a positive relevance and sensitivity between LER and evaluated standard deviation of pitch. Therefore, LER can be used as an aided pre-evaluation parameter for the pitch calculation method, such as the gravity center method or the zero-crossing points method. The optimization of the nano-grating evaluation method helps to obtain the accurate pitch value with fewer measurements and provide a comprehensive characterization of pitch standards.

Keywords: Pitch evaluation, nano-grating standard, line edge roughness, atomic force microscope.

\section{INTRODUCTION}

Lateral pitch standards, such as one-dimensional or two dimensional nano-gratings, are widely used as transfer standards to calibrate the nonlinearity or image magnification for all kinds of microscopes [1]. Many nanofabrication methods have been utilized to fabricate lateral pitch structures, including e-beam lithography, multilayer gratings [2], [3], atom lithography [4]-[6], atom-based rulers [7]-[9], and so on. Before the nanoscale pitch structures can be used as transfer standards, pitch uncertainties need to be measured with metrological AFMs and estimated with the effective pitch evaluation methods [10].

Effective pitch evaluation method is one of the key factors to lower the calculated pitch uncertainty, thereby increasing the calibration accuracy [11], [12]. The Gravity Center (GC) method [1], [13], [14], Zero Cross Points (ZCP) method [11] and Fourier Transform (FT) method [1], [15], [16] are the most commonly used pitch evaluation methods for one/two dimensional nano-grating standards. As a kind of pitch calculation method based on the real nano-grating profiles, the uncertainty of measurement results with GC or ZCP method relies on the selection of effective profiles. Therefore, optimization of effective profile selection for GC or ZCP methods offers a new way to increase the calibration accuracy of pitch standards.

Normally, line edge roughness (LER) refers to the randomly varied edges of critical dimensions (CD) of grate patterns [17]. Previous study has noted the importance of LER as a critical quality attribute of the pitch: a reference line with lower LER can achieve the same accuracy with fewer measurements [18]. Motivated by this concept, in this paper, we have introduced LER as a reference evaluation parameter for the pitch evaluation of one-dimensional self-traceable $\mathrm{Cr}$ atom lithography nano-gratings. The analysis shows that there is a positive correlation between LER and evaluated standard deviation of pitch of AFM measurement data, which provides key evidence for the effective profile selection during the pitch evaluation process and further increases the pitch calibration accuracy.

\section{THEORIES}

\section{A. Pitch evaluation method with LER}

Two profile-based methods are utilized in this paper: GC method and ZCP method, which are illustrated in Fig.1.a). First, a threshold line is set to divide the nano-grating profiles into two parts. For the GC method [1], [11]-[13], the geometrical center above the threshold line is treated as the gravity center; the distance of two neighboring gravity centers is defined as the pitch. For the ZCP method [11], [12], zero cross points are the intersection points between the threshold line and profile curves of the nano-grating pattern. The middle point of a pair of zero crossing points on each nano-grating pattern is treated as the geometric center point. Then the pitch values of the ZCP method are defined as the distance between neighboring middle points.

The profile cutting proportion (p), which is the profile below the threshold line, plays a critical role in pitch uncertainty estimation. Generally, the profile cutting 
proportion is set to be $50 \%$ of the peak to valley height (PTVH) as a tradition, but whether $50 \%$ is the best or not has not been elucidated clearly yet. If we expand the threshold line to a plane at the same height, then we get intersecting lines along the nano-grating edges, as demonstrated in Fig.1.b).

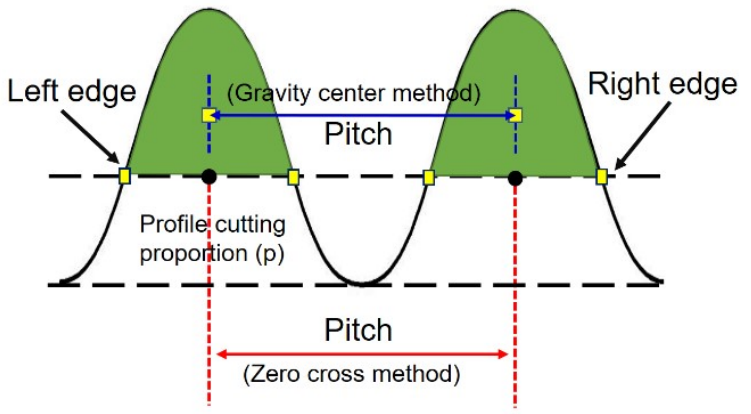

a)

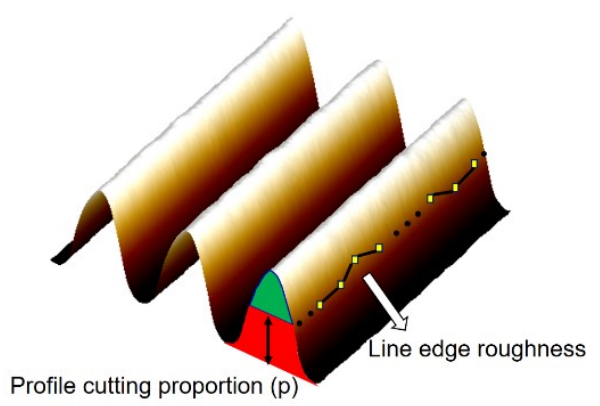

b)

Fig.1. a) Schematic of the Gravity Center method and the Zero Cross Method; b) Definition of line edge roughness of $\mathrm{Cr}$ atom lithography nano-gratings.

The LER describes the variation of the crossing points, which will provide effective information to select the best profile cutting proportion and, thereby, minimize the evaluated standard deviation of pitch. The average edge and the standard deviation $(\sigma)$ of the line edge are defined as follows [18]:

$$
\left\{\begin{array}{l}
\bar{x}=\frac{\left(\sum_{i=1}^{N} x_{i}\right)}{N} \\
3 \sigma=3 \sqrt{\frac{\sum_{i=1}^{N} \Delta x_{i}^{2}}{N-1}}=3 \sqrt{\frac{\sum_{i=1}^{N}\left(x_{i}-\bar{x}\right)^{2}}{N-1}}
\end{array}\right.
$$

Where $X_{i}$ is the position measured of the $i$ th point along the line edge. Normally, LER must be reported as $3 \sigma$ of the total, and in our case, we will investigate the LER of both edges over a length of L, and every time $\mathrm{L}$ is divided into 20 spacings apart. Then we obtained 21 measured positions to calculate the LER. The length $\mathrm{L}$ is assigned to be $0.5 \mathrm{P}, 1 \mathrm{P}$, $2 \mathrm{P}, 3 \mathrm{P}, 4 \mathrm{P}$, respectively, where $\mathrm{P}$ is the nominal pitch $(212.8 \mathrm{~nm})$.

\section{B. Cr atom lithography nano-gratings}

The nano-gratings used here are fabricated by laser focused $\mathrm{Cr}$ atomic deposition [4], [5], [19], i.e., the so-called Cr atom lithography nano-gratings. The detail experimental setup is described elsewhere [20], [21]. During the Cr nano-grating fabrication process, collimated $\mathrm{Cr}$ atoms are focused to the nodes or antinodes of a standing wave grazing across the substrate surface. So, the period of the $\mathrm{Cr}$ nano-gratings $(212.8 \mathrm{~nm})$ is directly traceable to the half of the laser light wavelength, which is strictly locked to specified atomic level transitions. In this way, the $\mathrm{Cr}$ atom lithography nanogratings can be used as self-traceable calibration length standard in nanotechnology.

The profile of $\mathrm{Cr}$ nano-gratings has an advantage of reducing the image distortion caused by the tip effect, which offers great convenience for the LER analysis. The images obtained by the AFM normally are a combination of tip geometry and sample surface. The linewidth is normally broadened due to the resulting dilation of the tip. Fig.2.a) is the cross section TEM image of $\mathrm{Cr}$ atom lithography nanogratings, the height to width ratio is relatively low, so the measured profile can reduce the tip effect and reveal the real profile to the full extent, as illustrated in Fig.2.b).

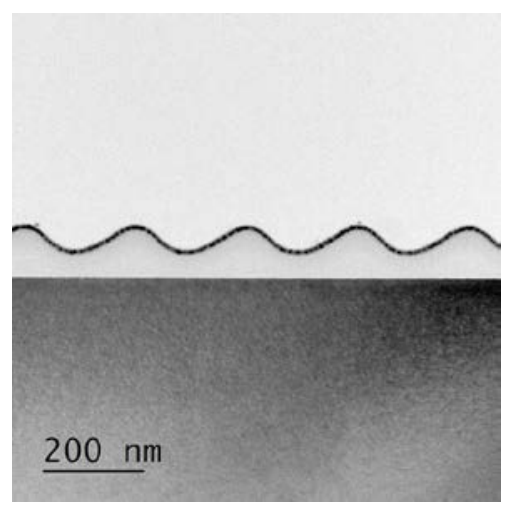

a)

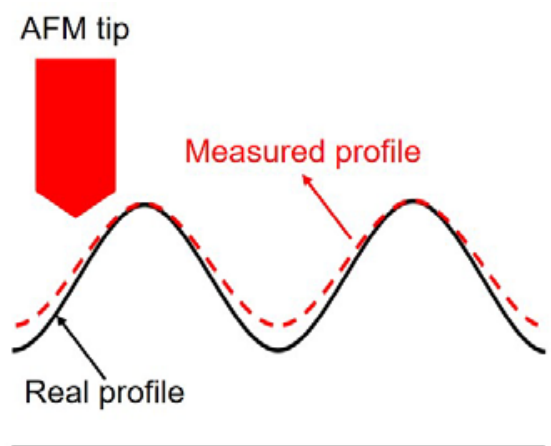

b)

Fig.2. a) Cross section TEM image of Cr nano-gratings; b) The discrepancy between measured profile and real profile of AFM measurement for $\mathrm{Cr}$ nano-gratings. 


\section{LINE EDGE ROUGHNESS ANALYSIS}

\section{A. Line edge roughness of $\mathrm{Cr}$ nano-gratings}

Fig..3 shows the AFM image and grating profile of Cr nanogratings used for the line edge roughness analysis. The selected PTVH is around $50 \mathrm{~nm}$ with very smooth and uniform parallel lines in Fig.3.a) and Fig.3.b). The original pixel size of the image is $488 \times 450$, we did the data interpolation and low pass filter to eliminate the imaging noise [1]. As mentioned before, the LER is calculated over left and right edges of a length $\mathrm{L}$ which is divided into 20 spacings apart (Fig.3.a)). The LER analysis results of this AFM image are typical and representative in similar image calculations.

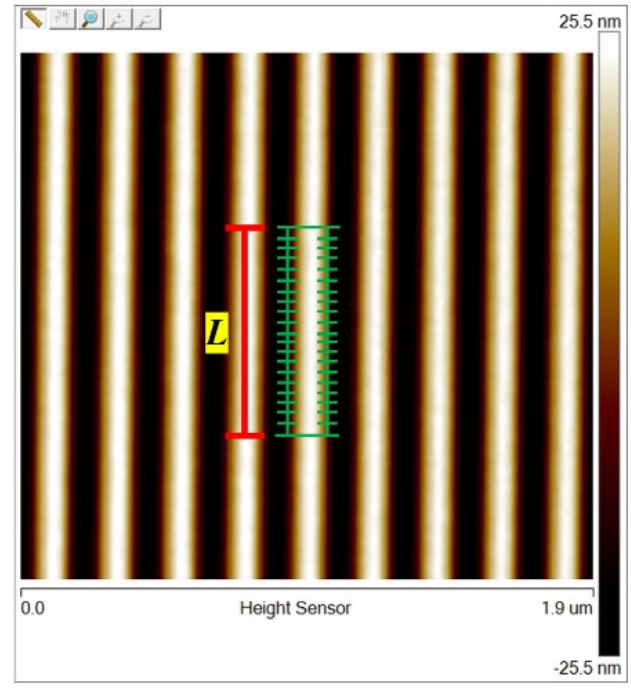

a)

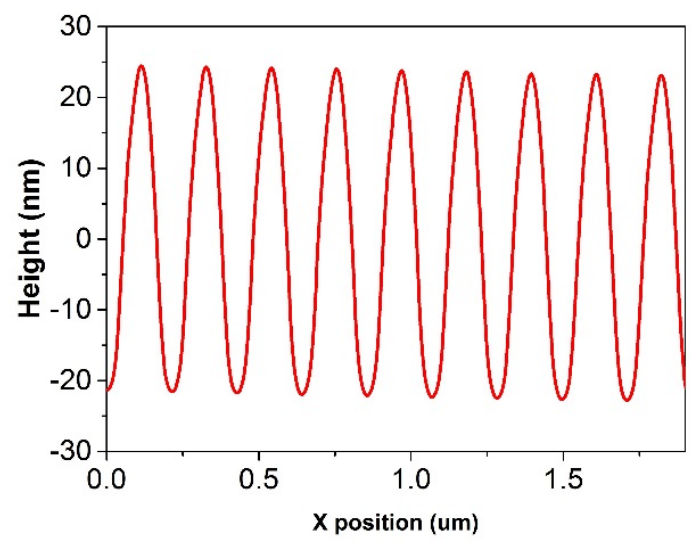

b)

Fig.3. a) AFM image of Cr nano-gratings $(1.9 \mu \mathrm{m} \times 1.75 \mu \mathrm{m})$; b) AFM profile of $\mathrm{Cr}$ nano-gratings with a peak to valley height of around $50 \mathrm{~nm}$.

Fig.4.a) shows the $3 \sigma$ LER of left and right edges as a function of profile cutting proportion (p), and the calculation length $\mathrm{L}$ of the LER is $\mathrm{P}(212.8 \mathrm{~nm})$. Here the profile cutting proportion (p) is the profile below the threshold line in Fig.1.a). It is obvious that the LER keeps at a very low level (below $1 \mathrm{~nm}$ ) when p ranges from 0.2 to 0.6 and the minimum
LER appears at $\mathrm{p}=0.3$ of these discrete value assignments, which verified the speculation about the best $p$ selection. The LER will increase when p decreases to 0.1 due to increased randomness of the laser focused $\mathrm{Cr}$ structures at the top. The same trend appears when $\mathrm{p}$ increases to 0.9 , together with the increased discrepancy between left LER and right LER, which are highly possibly caused by the AFM scanning distortion induced by the tip effect. In addition, it should be noted that the LER of $\mathrm{p}<0.5$ of left and right edges are almost identical even with a slightly asymmetric structure, which may be an evidence to explain why the self-traceable $\mathrm{Cr}$ nanogratings have an advantage of low uncertainty.

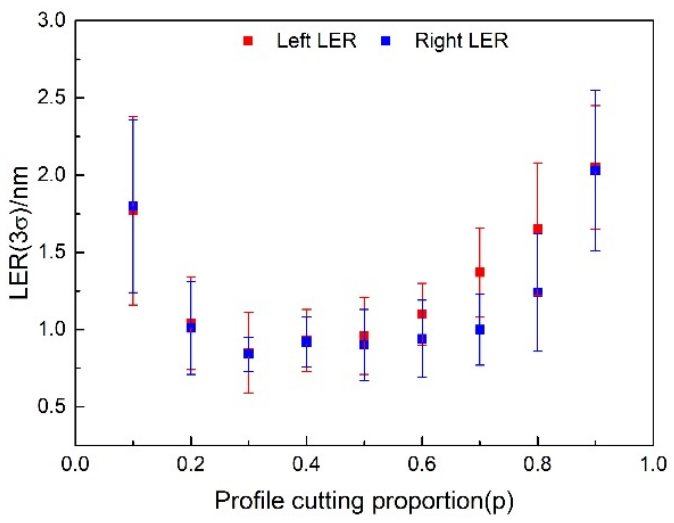

a)

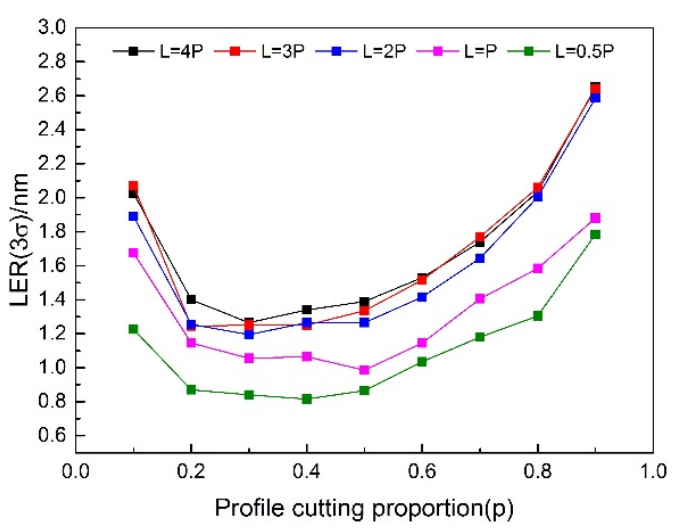

b)

Fig.4. a) Left and Right LER as a function of profile cutting proportion; b) Average LER as a function of profile cutting proportion over different calculation length (0.5P 4P).

Fig.4.b) demonstrates the average LER of left and right edges as a function of profile cutting proportion over different calculation length ranging from $0.5 \mathrm{P}$ to $4 \mathrm{P}$. The similar distribution phenomenon shows up over different calculation length in Fig.4.a). As the calculation length increases from $0.5 \mathrm{P}$ to $2 \mathrm{P}$, the average LER rises gradually; but after the length exceeds $2 \mathrm{P}$ to $4 \mathrm{P}$, the average LER keeps stable without obvious increase. This indicates that a calculation length of $2 \mathrm{P}(425.6 \mathrm{~nm})$ is long enough to evaluate the maximum LER level of the laser focused $\mathrm{Cr}$ atomic nanogratings. 
B. The relationship between line edge roughness and evaluated standard deviation of pitch

Next, we examined the relationship between lined edge roughness and the evaluated standard deviation of pitch. The AFM images we calculated were acquired by a commercial AFM (Bruker, Dimension Edge), which is non-metrological. As we introduced in the context, the $\mathrm{Cr}$ atom lithography nano-grating used here is self-traceable, its pitch is expected to be $212.8 \mathrm{~nm}$. Jabez. J. McClelland has even examined the average pitch of $\mathrm{Cr}$ nano-gratings by optical diffraction method, which turned out to be $212.7777 \pm 0.0069 \mathrm{~nm}$, with a relative uncertainty of a few times $10^{-5}$ [22]. Therefore, we corrected the calculated average pitch to $212.8 \mathrm{~nm}$ and got the evaluated standard deviation of pitch by a correction coefficient.

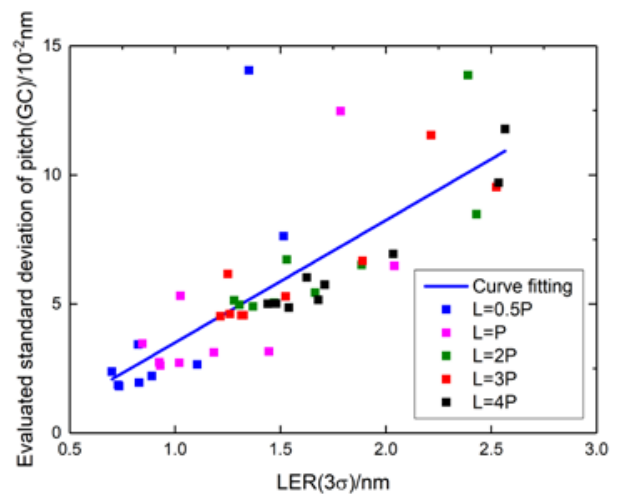

a)

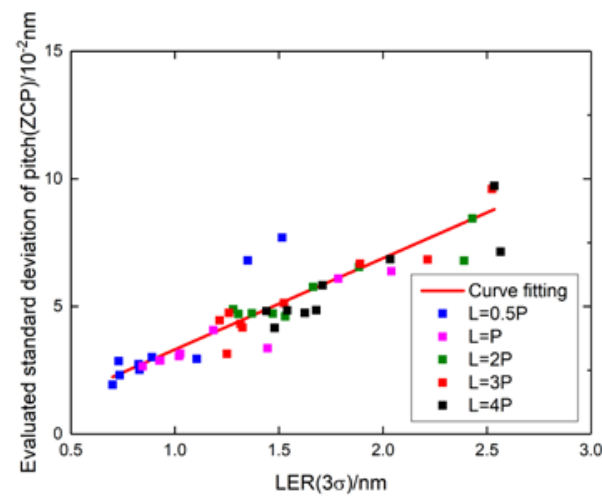

b)

Fig.5. Relationship between LER with evaluated standard deviations of pitch calculated by a) Gravity center method; b) Zero cross points method.

Fig.5. shows the corresponding evaluated standard deviations of pitch under every LER condition over different calculation lengths varying from $0.5 \mathrm{P}$ to $4 \mathrm{P}$. Fig.5.a) and Fig.5.b) are the results of the gravity center method and the zero-crossing points method, respectively. From both figures, it is obvious that there is a positive correlation between LER and evaluated standard deviation of pitch, and the relationship tends to be linear. Compared with the ZCP method, the sensitivity scale of evaluated standard deviation of pitch on LER based on the GC method is a little bit higher. The reason for this discrepancy is the difference between GC and ZCP method. In the ZCP method, only the LER information at the reference line are involved in the pitch calculation, while in the GC method all the LER information above the reference line are contained inside. Though some of the lined edge randomness is neutralized because of the nano-gratings' symmetry, the top part of the nano-grating (for example, $10 \%$ of the top profile) has lower symmetry and higher LER as demonstrated in Fig.4.a). The relationship of LER and evaluated standard deviation of pitch determines that LER can be used as an aided pre-evaluated parameter for the pitch evaluation method, which offers a more comprehensive evaluation result for the pitch standards.

\section{Pitch evaluation method optimization}

The fundamental pitch distance, the pitch uniformity, the quality of the LER and the accuracy of the certified pitch value, and the traceability are the most critical qualities which attribute a pitch standard [18]. From the analysis of selftraceable $\mathrm{Cr}$ nano-gratings, there is a strong positive correlation between LER and evaluated standard deviation of pitch. Based on the relevance of these two parameters, it is suggested to introduce LER calculation as an aided preevaluation parameter for the pitch calculation method, especially for best profile cutting proportion selection during GC or ZCP method. This not only helps to obtain the accurate pitch value with fewer averaging sample measurements, but also helps to extract more accurate pitch evaluation information with the same experimental data. At the same time, together with evaluated standard deviation of pitch, LER analysis offers a comprehensive evaluation about the critical qualities of a pitch, such as the accuracy and the uniformity characteristics.

\section{CONCLUSIONS}

In this paper, a LER analysis of self-traceable $\mathrm{Cr}$ nanogratings was conducted to show the relevance between the LER and evaluated standard deviation of pitch in the pitch evaluation method (i.e. gravity center method and zero cross points method). The results demonstrate a positive correlation between LER and the evaluated standard deviation of pitch, which indicates that LER can be used as an aided preevaluation parameter for pitch calculation method, especially for effective profile cutting proportion selection for GC or ZCP method. The optimization of the nano-grating pitch evaluation method based on LER would offer convenience to obtain the accurate pitch value with fewer measurements and provide a comprehensive evaluation about the critical qualities of pitch standards.

\section{ACKNOWLEDGEMENT}

This work is supported by National Natural Science Foundation of China (Grant No. 51475335, 51705369), National Key Research and Development Program of China (Grant No.2016YFA0200902), National Key Scientific Instrument and Equipment Development Projects of China (No.2014YQ090709) and China Postdoctoral Science Foundation (No.2017M611613). 


\section{REFERENCES}

[1] Dai, G., Koenders, L., Pohlenz, F., Dziomba, T., Danzebrink, H. (2005). Accurate and traceable calibration of one-dimensional gratings. Measurement Science and Technology, 16 (6), 1241-1249.

[2] Misumi, I., Dai, G., Lu, M., Sato, O., Sugawara, K., Gonda, S., Takatsuji, T., Danzebrink, H., Koenders, L. (2010). Bilateral comparison of $25 \mathrm{~nm}$ pitch nanometric lateral scales for metrological scanning probe microscopes. Measurement Science and Technology, 21 (3), 035105.

[3] Xingrui, W., Zhao, Y., Liu, J., Chen, J., Li, T., Xinbin, C. (2016). Fabrication and characterization of onedimensional multilayer gratings for nanoscale microscope calibration. In Nanoengineering: Fabrication, Properties, Optics and Devices XIII, 2831 August 2016, San Diego, US. SPIE 9927.

[4] McClelland, J., Scholten, R., Palm, E., Celotta, R. (1993). Laser-focused atomic deposition. Science, 262 (5135), 877-880.

[5] Celotta, R., McClelland, J. (1994). Method of fabricating laser controlled nanolithography. Patent US5360764 A.

[6] Xiao, D., Tongbao, L., Lihua, L., Yan, M., Rui, M., Junjing, W., Yuan, L. (2014). Fabrication and measurement of traceable pitch standard with a big area at trans-scale. Chinese Physics B, 23 (9), 143-147.

[7] Dai, G., Heidelmann, M., Kübel, C., Prang, R., Fluegge, J., Bosse, H. (2013). Reference nano-dimensional metrology by scanning transmission electron microscopy. Measurement Science and Technology, 24 (8), 085001.

[8] Li, K. (2011). Nanofabrication on engineered silicon (100) surfaces using scanning probe microscopy. Unpublished doctoral dissertation, University of Maryland, US.

[9] Ballard, J.B., Dick, D.D., McDonnell, S.J., Bischof, M., Fu, J., Owen, J.H., Owen, W.R., Alexander, J.D., Jaeger, D.L., Namboodiri, P. (2015). Atomically traceable nanostructure fabrication. Journal of Visualized Experiments Jove, 101, e52900.

[10] Korpelainen, V. (2014). Traceability for nanometre scale measurements: Atomic force microscopes in dimensional nanometrology. Unpublished doctoral dissertation, University of Helsinki, Finland.

[11] Huang, Q., Gonda, S., Misumi, I., Keem, T., Kurosawa, T. (2006). Research on pitch analysis methods for calibration of one-dimensional grating standard based on nanometrological AFM. In Third International Symposium on Precision Mechanical Measurements, 25 August 2006, Urumqi, China. SPIE 6280.
[12] Huang, Q., Misumi, I., Gonda, S., Sato, O., Kurosawa, T. (2006). Pitch calibration of one-dimensional grating standard by tapping mode nanometrological atomic force microscope. In Sixth International Symposium on Instrumentation and Control Technology: Signal Analysis, Measurement Theory, Photo-Electronic technology, and Artificial Intelligence, 13-16 October 2006, Beijing, China. SPIE 6357.

[13] Misumi, I., Gonda, S., Kurosawa, T., Takamasu, K. (2003). Uncertainty in pitch measurements of onedimensional grating standards using a nanometrological atomic force microscope. Measurement Science and Technology, 14 (4), 463-471.

[14] Dai, G., Pohlenz, F., Dziomba, T., Xu, M., Diener, A., Koenders, L., Danzebrink, H. (2007). Accurate and traceable calibration of two-dimensional gratings. Measurement Science and Technology, 18 (2), 415-421.

[15] Li, C., Yang, S., Wang, C., Jiang, Z. (2016). EEMD based pitch evaluation method for accurate grating measurement by AFM. Applied Surface Science, 380, 274-280.

[16] Chen, X., Koenders, L. (2014). A novel pitch evaluation of one-dimensional gratings based on a crosscorrelation filter. Measurement Science and Technology, 25 (4), 044007.

[17] Shin, C. (2016). Variation-Aware Advanced CMOS Devices and SRAM. Springer, 56.

[18] Tortonese, M., Prochazka, J., Konicek, P., Schneir, J., Smith, I.R. (2002). 100-nm-pitch standard characterization for metrology applications. In Metrology, Inspection, and Process Control for Microlithography XVI, 3-6 March 2002, Santa Clara, Canada. SPIE 4689, 558-564.

[19] Lihua, L., Yuan, L., Xiao, D., Guofang, F., Xiaoyu, C., Xinbin, C., Junjing, W., Gang, L., Tongbao, L. (2015). Laser-focused $\mathrm{Cr}$ atomic deposition pitch standard as a reference standard. Sensors and Actuators A: Physical, 222, 184-193.

[20] Xiao, D., Yan, M., Pingping, Z., Wanjing, Z., Sheng, C., Shengwei, X., Tongbao, L. (2012). Investigation of shadow effect in laser-focused atomic deposition. Applied Surface Science, 261, 464-469.

[21] Lihua, L., Yuan, L., Guofang, F., Junjing, W., Xiao, D., Xiaoyu, C., Tongbao, L. (2014). Nano-traceability study of a $\mathrm{Cr}$ standard grating fabricated by laserfocused atomic deposition. Chinese Physics Letters, 31 (7), 46-49.

[22] McClelland, J.J., Anderson, W.R., Bradley, C.C., Walkiewicz, M., Celotta, R.J., Jurdik, E., Deslattes, R.D. (2003). Accuracy of nanoscale pitch standards fabricated by laser-focused atomic deposition. Journal of Research-National Institute of Standards and Technology, 108 (2), 99-113.

Received July 30, 2017. Accepted November 13, 2017. 